\title{
REFLEXIONES CRÍTICAS SOBRE LOS ESTUDIOS DE POBREZA EN COSTA RICA
}

\author{
MAYELA CUBILLO MORA \\ Universidad de Costa Rica. \\ mayela.cubillo@ucr.ac.cr
}

\section{RESUMEN}

El propósito de este artículo es describir el campo simbólico de fuerzas alrededor del concepto de pobreza que logran imponer significados, y asignarlos como legítimos. Se revisarán los discursos sociales, imaginarios y paradigmas sobre la pobreza, que han tejido tanto los académicos como los organismos nacionales e internacionales que trabajan en la temática. La formulación de las políticas sociales, las herramientas e instrumentos para "definir" y "medir" la pobreza y la forma de combatirla, y el trato mismo que los funcionarios públicos dan a las personas en condición de pobreza, dependen de la concepción que hay detrás de esos discursos y paradigmas.

PALABRAS CLAVE: POBREZA, IMAGINARIO, DISCURSO, PARADIGMA.

\section{ABSTRACT}

The purpose of this article is to describe the symbolic field around the concept of poverty (social and imaginary speeches, and paradigms), that have woven both the academics as national and international agencies working in the theme. The formulation of social policies, tools and instruments for "define" and "measure" and the poverty and how to fight it and the treatment that the public workers give to the poor people depend of the conception behind those speeches and paradigms.
KEY WORDS: POVERTY, SOCIAL SPEECHES, IMAGINARY, PARADIGMS.

\section{INTRODUCCIÓN}

Mucho se ha discutido desde la academia sobre el origen, desarrollo y evolución del fenómeno de la pobreza. El análisis del tema se encuentra influenciado o determinado por las perspectivas ideológicas, los discursos sociopolíticos, los imaginarios sociales y las experiencias concretas de los actores sociales que trabajan alrededor del tema. No obstante, la diversidad de visiones con las que se aborda la cuestión, existe una realidad concreta y palpable, y es que la pobreza existe, según cifras del Instituto Nacional de Estadísticas y Censos (INEC, 2010), al mes de noviembre de 2010, el 19,4\% de los costarricenses la padecen, es decir alrededor de 750000 personas. Lo real, concreto es que es un problema de gran magnitud, el cual debe ser atendido.

Cabe señalar que este es un artículo eminentemente descriptivo, y es irremediablemente provisional y polémico debido al enorme corpus de los estudios relacionados con la comprensión de la pobreza a escala nacional y regional, a las contribuciones que esta multitud de investigaciones ha producido en varios planos y a la complejidad temática, teórica y metodológica implicada por esos esfuerzos. 
En todo caso, este trabajo aspira a aportar una propuesta de lectura que contribuya a valorar la necesidad de abrir paso a nuevos enfoques teóricos y prácticos capaces de explicar por qué no se han cumplido las promesas de crecimiento económico, expansión del empleo y reducción de la pobreza y la desigualdad, realizadas en el marco de los procesos de estabilización y ajuste económico en América Latina.

Las categorías conceptuales utilizadas para el artículo, provienes de los ámbitos de la filosofia de la ciencia, la sociología. Los conceptos de campo simbólico, imaginario, discurso y paradigma fueron desarrollados en los ámbitos de la filosofía de la ciencia y la arqueología del saber (Castoriadis 1986; Kuhn 1987; Foucault 1984, Bourdieu 1991).

\section{Campo simbólico, imaginarios sociales, discursos sociales y paradigmas sobre la pobreza}

Para Bourdieu (1991), el campo simbólico se instaura a partir de la imposición de significados legítimos, ilegitimando a otros no convenientes, contrarios, dominando a la otra parte de la relación. Para el autor, este campo de fuerzas precisó de pasar de la fuerza, de la violencia física a relaciones legítimas como son las puramente simbólicas: la religión, la cosmovisión que permiten hacer creer a los dominados que los dominadores tienen una autoridad legítima para ejercer la autoridad.

Para Castoriadis (1986), los procesos simbólicos como los imaginarios sociales permiten a una sociedad que se asigne puntos de referencia para implantarse en el espacio y el tiempo, para hacer posible la comunicación entre sus miembros y para situarse en relación con las otras sociedades. Estos puntos de referencia simbólicos, son empleados en la vida cotidiana por los actores sociales o los individuos, y definen en una situación particular qué es real, qué tiene o no sentido, y a partir de ahí, interpretan, valoran o se comportan de determinada forma.

Los discursos sociales son un orden simbólico que permite a todos los miembros de una sociedad que fueron socializados en ella hablar y actuar juntos. Este conjunto de construcciones circulan en una sociedad con eficacia para la producción/reproducción de representaciones perceptuales y de interpretaciones conceptuales o valorativas, y que según Foucault (1984) se constituyen en instrumento de poder e ideologia.

Con respecto a los paradigmas Thomas Kuhn (1987) estableció que las prácticas intelectuales se organizan alrededor de paradigmas y estos son mucho más formalizados que los discursos. Los paradigmas científicos pueden conceptualizarse como representaciones básicas idénticas y opuestas, compartidas o descalificadas no solo por las comunidades de científicos sociales, sino por los funcionarios de instituciones nacionales e internacionales. Dichos paradigmas definen los temas de las agendas nacionales e institucionales, su abordaje teórico y metodológico, los instrumentos y los parámetros para la toma de decisiones, así como para la formulación y ejecución de las políticas públicas.

Esping-Andersen (1987), establece una vinculación entre paradigmas y discursos, no así con los imaginarios. Sin embargo, se realiza en la tabla 1 una vinculación entre los imaginarios, los discursos y los paradigmas. 


\section{TABLA 1}

\section{Vinculación entre imaginarios, discursos sociopolíticos y paradigmas}

\begin{tabular}{|c|c|c|c|}
\hline Primer Imaginario & $\begin{array}{l}\text { Discurso sociopolítico } \\
\text { correspondiente }\end{array}$ & $\begin{array}{c}\text { Paradigma correspon- } \\
\text { diente }\end{array}$ & Comentario \\
\hline $\begin{array}{l}\text { Siglos XVIII y XIX. } \\
\text { La pobreza es consecuencia } \\
\text { de incapacidades personales y } \\
\text { morales. Como los pobres ne- } \\
\text { cesitan disciplina, solo quienes } \\
\text { enfrentan situaciones extremas } \\
\text { obtendrán algún tipo de apoyo } \\
\text { si están dispuestos a reformarse } \\
\text { y a buscar sus medios de subsis- } \\
\text { tencia en el mercado. } \\
\text { Se combate la pobreza median- } \\
\text { te la filantropía, caridad ecle- } \\
\text { siástica y privada o mediante } \\
\text { leyes que castigan la vagancia. }\end{array}$ & $\begin{array}{l}\text { El discurso neoliberal. } \\
\text { La idea central de este dis- } \\
\text { curso es que en la socie- } \\
\text { dad de mercado al buscar } \\
\text { el bienestar individual se } \\
\text { conseguirá el bienestar co- } \\
\text { lectivo. } \\
\text { Este enfoque plantea dejar } \\
\text { a la libre las fuerzas del mer- } \\
\text { cado, ofreciendo alivio solo } \\
\text { a quienes por edad, enfer- } \\
\text { medad, pobreza extrema o } \\
\text { vulnerabilidad son incapa- } \\
\text { ces de obtener ingresos. } \\
\text { Se separa al pobre y exclui- } \\
\text { do del status de ciudadanía. }\end{array}$ & $\begin{array}{l}\text { El paradigma residual. } \\
\text { Establece al mercado como } \\
\text { el centro de la vida social, } \\
\text { reduce el rol del Estado a } \\
\text { garantizar el buen funcio- } \\
\text { namiento de la macroeco- } \\
\text { nomía, y a la atención de } \\
\text { los riesgos no rentables, } \\
\text { transfiriendo el manejo del } \\
\text { resto de los seguros socia- } \\
\text { les a los individuos. } \\
\text { Ataca los derechos y titula- } \\
\text { ridades sociales y promue- } \\
\text { ve soluciones de mercado } \\
\text { para los problemas sociales. }\end{array}$ & $\begin{array}{l}\text { Al escuchar a algunos de } \\
\text { nuestros políticos, comuni- } \\
\text { cadores e incluso técnicos, } \\
\text { su discurso es que se trate } \\
\text { de evitar transferir recursos } \\
\text { públicos a quienes se perci- } \\
\text { be "pueden" estar intentando } \\
\text { evitar un empleo honesto. } \\
\text { Se pregona las transferencias } \\
\text { condicionadas, para no dar } \\
\text { algo a cambio de nada. }\end{array}$ \\
\hline
\end{tabular}

\begin{tabular}{|c|c|c|c|}
\hline Segundo Imaginario & $\begin{array}{l}\text { Discurso sociopolítico } \\
\text { correspondiente }\end{array}$ & $\begin{array}{c}\text { Paradigma } \\
\text { correspondiente }\end{array}$ & $\begin{array}{c}\text { Comentario } \\
\text { personal }\end{array}$ \\
\hline $\begin{array}{l}\text { Finales siglo XIX y principios si- } \\
\text { glo XX } \\
\text { Son pobres quienes no están } \\
\text { integrados a las diferentes for- } \\
\text { mas de organización familiar, } \\
\text { gremial o corporativa que les } \\
\text { permita intercambiar bienestar } \\
\text { y articularse al Estado. }\end{array}$ & $\begin{array}{l}\text { Discurso conservador } \\
\text { Distingue a los pobres de } \\
\text { los trabajadores formales, a } \\
\text { través de la administración } \\
\text { diferenciada de la seguridad } \\
\text { laboral. } \\
\text { Esta diferenciación supone } \\
\text { la construcción de sectores } \\
\text { protegidos institucional y } \\
\text { normativamente y de otros } \\
\text { dejados en situación de po- } \\
\text { breza. }\end{array}$ & $\begin{array}{l}\text { Establece la obligatoriedad } \\
\text { del seguro social para los } \\
\text { trabajadores formales y es- } \\
\text { quemas asistenciales para } \\
\text { aquellos que no cuentan } \\
\text { con un empleo formal. } \\
\text { Establece riesgos por gru- } \\
\text { pos ocupacionales, y desa- } \\
\text { rrolla derechos diferencia- } \\
\text { les. }\end{array}$ & $\begin{array}{l}\text { Las sociedades que adopten } \\
\text { este enfoque favorecerán la } \\
\text { reproducción de la pobreza } \\
\text { y la exclusión sistemática de } \\
\text { algunos grupos y categorías } \\
\text { sociales en el esquema de } \\
\text { protección del Estado. }\end{array}$ \\
\hline
\end{tabular}




\begin{tabular}{|c|c|c|c|}
\hline Tercer Imaginario & $\begin{array}{l}\text { Discurso sociopolítico } \\
\text { correspondiente }\end{array}$ & $\begin{array}{c}\text { Paradigma } \\
\text { correspondiente }\end{array}$ & $\begin{array}{c}\text { Comentario } \\
\text { personal }\end{array}$ \\
\hline $\begin{array}{l}\text { Siglo XIX y XX } \\
\text { La pobreza es concebida como } \\
\text { producto del funcionamiento } \\
\text { del mercado y de los privilegios } \\
\text { de grupos sociales organizados. } \\
\text { La pobreza no es responsabi- } \\
\text { lidad de los individuos, sino } \\
\text { de sus grupos de pertenencia, } \\
\text { los empleadores, el Estado y el } \\
\text { conjunto de la sociedad a tra- } \\
\text { vés de impuestos de aplicación } \\
\text { general. } \\
\text { Se combate a través de la fija- } \\
\text { ción de una matriz de derechos } \\
\text { sociales para cada individuo } \\
\text { que les garanticen un nivel mí- } \\
\text { nimo de bienestar o calidad de } \\
\text { vida. }\end{array}$ & $\begin{array}{l}\text { Discurso social demócrata } \\
\text { Este enfoque se remite al } \\
\text { establecimiento de están- } \\
\text { dares de bienestar, en tér- } \\
\text { minos culturales y sociales. } \\
\text { La idea central planteada } \\
\text { por primera vez por T. H. } \\
\text { Marshall (1964) es garanti- } \\
\text { zar a los pobres el derecho, } \\
\text { las titularidades con las que } \\
\text { ya cuentan el resto de los } \\
\text { individuos, a compartir la } \\
\text { herencia social y a ser acep- } \\
\text { tados como miembros ple- } \\
\text { nos de la sociedad. } \\
\text { Esta posición implica asumir } \\
\text { que ofrecer la posibilidad } \\
\text { de una ciudadanía social } \\
\text { plena a los pobres, solo es } \\
\text { posible limitando la libertad } \\
\text { de mercado. }\end{array}$ & $\begin{array}{l}\text { Universalista } \\
\text { Plantea que si el Estado } \\
\text { cubre simultáneamente la } \\
\text { mayoría de los riesgos so- } \\
\text { ciales, (por medio de los se- } \\
\text { guros) y al conjunto de los } \\
\text { ciudadanos, estableciendo } \\
\text { derechos sociales exigibles } \\
\text { individualmente, se favo- } \\
\text { rece la reducción de la po- } \\
\text { breza y el desarrollo de la } \\
\text { ciudadanía y los derechos y } \\
\text { titularidades sociales. }\end{array}$ & $\begin{array}{l}\text { Para el caso costarricense lo } \\
\text { que ocurrió realmente fue que } \\
\text { partidos políticos reformado- } \\
\text { res se pusieron de acuerdo en } \\
\text { un modelo de país solidario y } \\
\text { de bienestar, y de un Estado } \\
\text { que tenía que tutelar todos } \\
\text { esos derechos y administrar } \\
\text { muchos de ellos. } \\
\text { Sin embargo, el énfasis en lo } \\
\text { estatal y lo normativo ha crea- } \\
\text { do muchas organizaciones } \\
\text { para la tutela y administración } \\
\text { de tales derechos, dando lugar } \\
\text { a clientelismos político-electo- } \\
\text { rales y personalistas que han, } \\
\text { a su vez, producido grandes } \\
\text { desviaciones en el sistema } \\
\text { político-institucional. }\end{array}$ \\
\hline
\end{tabular}

Fuente: elaboración propia, a partir de Esping-Andersen (1987). Los comentarios de la última columna son aporte de la autora.

\section{El campo simbólico sobre el concepto de pobreza}

Se ha procedido en esta parte a realizar una agrupación y clasificación de los conceptos de pobreza, que si bien puede resultar arbitraria para algunos, ayuda a realizar una bien sustentada y necesaria revisión histórica del concepto, desde las diferentes fuerzas que han trabajado el término.

\section{Desde el enfoque}

de las necesidades básicas

El planteamiento original de Rowntree (1901) es entender la pobreza, como un mínimo de bienes que debe tener una persona o grupo para encontrarse en una situación digna de vida, si no se da hay una pérdida de bienestar.
A esta primera definición (Townsend, 1993) realiza una crítica en el sentido de que las personas son vistas como organismos biológicos que requieren la restitución de sus fuentes de energía, pero dejan de lado que son seres sociales, participantes de relaciones sociales complejas, y también son productores de bienes para los cuales dependen de instalaciones y servicios públicos que son producto de un esfuerzo colectivo.

A partir de la propuesta de Townsend, se aceptó una segunda conceptualización de la pobreza: la de las necesidades básicas insatisfechas, que incluye dos elementos a destacar; primero, los requerimientos mínimos de consumo privado de una familia (alimentación, techo y vestido adecuados, así como cierto mobiliario y equipo doméstico). Segundo, los servicios esenciales provistos por y para la comunidad, como agua potable, servicios sanitarios, transporte público, 
servicios de atención a la salud, educación e instalaciones y centros culturales.

Ese enfoque tiene una gran influencia. Su principal representante es el Banco Mundial, y es el que se aplica en las mediciones y estudios anuales en instituciones como la CEPAL y el Instituto Nacional de Estadística y Censos de Costa Rica- INEC.

Desde el enfoque de las capacidades individuales/titularidades

Amartya Sen y Dréze (1989) concentran sus planteamientos sobre la pobreza en el tema de la construcción de capacidades y habilidades de los individuos. Para los autores, la privación y la brecha de acceso a recursos (hogar, tierra entre otros) reflejan al mismo tiempo, una brecha en las titularidades (entitlements), es decir no se trata de la ausencia de elementos básicos para la vida como bienes, sino de los derechos sobre éstas. Las titularidades permiten analizar cómo las estructuras sociales le permiten a los individuos acceder a un bien. Cualquier desigualdad en la estructura social genera un problema de capacidades.

\section{Desde el enfoque de insuficiencia} de medios para adquirir recursos

Se parte de que existe en la sociedad una desigual distribución de los ingresos, que crea grandes brechas entre las personas, y no les permite a los individuos acceder a recursos, y con consecuencia de la falta de ingresos, se dan brechas entre niveles de vida entre las personas. Se entiende que existe un nivel mínimo de vida razonable frente a los estándares de la sociedad. Anthony Atkinson y François Bourguignon (1999) son representantes de esta posición.

\section{Desde el enfoque de la privación relativa}

Este enfoque se basa en la idea de que el modo en que las personas y las familias perciben su estado de pobreza depende de su posición en la sociedad respecto a los demás. En otras palabras, el concepto de pobreza relativa presta atención a que un individuo puede ser más o menos pobre según cuanto tengan los demás. Como lo que tengan unos y otros varía conforme el ingreso medio de un país, y éste va creciendo, la pobreza se ve como una privación relativa en el sentido de que ella está relacionada con la disponibilidad de bienes y la modificación que va ocurriendo en las pautas de consumo, o sea, en la forma en que van evolucionando las necesidades. Entonces, la pobreza se ve como una privación relativa ligada a las variantes del ingreso medio. Sen (1984) argumenta con razones similares: que el concepto de pobreza relativa surge con el fin de tener una visión de la pobreza que tome en cuenta las nuevas necesidades de las familias y personas conforme transcurre el tiempo.

\section{Desde el enfoque marxista}

En la visión marxista la apropiación por una clase social (la capitalista) del excedente social o plusvalía, que otros crean (fuerza de trabajo en general, especialmente la asalariada) produce grandes desigualdades entre los grupos sociales, los cuales explican el bajo nivel de bienestar de los asalariados. Este problema es de estructura social y genera dificultades en los accesos a bienes por parte de los grupos que no son dueños de medios de producción.

\section{Desde el enfoque moral}

Desde una visión ética la posición normativa frente a la pobreza es vista como un Juicio Moral. Siguiendo a Cortina (2009) que plantea reconocer a la persona como valor absoluto, pensamos que en cada situación concreta, en que las relaciones sociales sean tales que una sola persona resulte dañada en sus derechos físicos o morales, tenemos un criterio para juzgarla como inmoral. Si hay pobres en un mundo en el que hay quienes derrochan, las relaciones sociales son inmorales. 


\section{Del concepto a la medición de pobreza}

Para distinguir a los pobres de los no pobres, los investigadores han construido los términos umbral o línea que permite distinguir operacional o empíricamente entre unos y otros, en una sociedad. Tales límites pueden estar expresados en términos monetarios (por ejemplo, el monto de ingreso mínimo requerido para tener acceso a los bienes de consumo que satisfagan las necesidades básicas para no caer en pobreza); o no monetarias (por ejemplo, el nivel mínimo de educación requerido para satisfacer la necesidad de educación).

\section{Medición según}

el concepto de subsistencia

El procedimiento de medición de la pobreza que más usan los técnicos es el ingreso de los hogares. Con respecto a este indicador, se calcula el monto del ingreso que le permite a la familia satisfacer las necesidades fundamentales. A partir de este monto se define la Línea de la Pobreza (ver CEPAL www.eclac.cl/deype/mecovi/ taller4.htm).

La investigación de los ingresos, se realiza mediante encuestas, que determinan si los hogares tienen ingresos suficientes para adquirir los bienes y servicios necesarios a fin de satisfacer las necesidades básicas. Estas necesidades se determinan a priori y luego se construye una canasta básica de bienes alimentarios y servicios que cumpla con los requisitos de satisfacción de tales necesidades. Una vez definida la canasta básica, se obtiene su costo valorando los artículos a precios de mercado. Este costo define la línea de pobreza. Si el ingreso del hogar se encuentra por debajo de la línea, el hogar es pobre.

Respecto a los bienes de alimentación se trata de conformar una canasta que satisfaga las necesidades básicas de nutrición, que combine alimentos que contengan los nutrientes necesarios de más bajo costo, respetando el patrón de alimentación observado en la población. Según Céspedes y Jiménez (2006), en el caso de Costa Rica, se establece para integrar la canasta básica una tabla calórica por sexo, edad, actividad física, estado fisiológico, se determina los alimentos a incluir y se constata por medio del índice de precios al consumidor, el costo de éstos.

Por su parte, para la construcción de la canasta básica de "otros bienes o servicios" se presentan dos alternativas. Una consiste en proceder de manera similar a la de los alimentos, e identificar expresamente los requerimientos mínimos de cada necesidad, como vivienda, vestuario, educación, transporte, entre otros- Una segunda vía es no intentar especificar en estos casos los requerimientos mínimos, sino simplemente utilizar la proporción observada de gasto en esos bienes dentro del gasto total de los hogares, en un grupo particular de la población.

En sus estudios, la CEPAL (1993) ha diseñado dos Líneas de la Pobreza: una en las áreas urbanas, y otra en las áreas rurales ( ver www.eclac.cl/deype/mecovi/docs/TALLER13/7.pdf)

\section{Medición según necesidades básicas insatisfechas}

Las necesidades consideradas básicas tomadas en consideración para determinar si una familia las enfrenta, depende de la información disponible propia de cada país. Existen ciertas necesidades que usualmente se incluyen bajo el concepto de necesidades básicas insatisfechas, en los estudios en Costa Rica. Ellas son: a) acceso a albergue digno: vivienda adecuada, sin hacinamiento, con calidad (materiales de construcción utilizados en piso, paredes y techo, así como con aspectos de privacidad e higiene, b) acceso a condiciones de higiene sanas: disponibilidad de abastecimiento permanente de agua potable y en cantidad suficiente para satisfacer las nece- 
sidades de alimentación e higiene y acceso a servicios sanitarios con sistema de aguas negras, c) acceso a conocimiento básico: niños y jóvenes deben tener acceso a una educación básica mínima, y d) acceso a otros bienes y servicios: recursos suficientes del jefe de hogar para que él y sus dependientes tengan una capacidad mínima de consumo.

El umbral elegido para cada necesidad corresponde a la mínima satisfacción posible de necesidades que sea compatible con una participación adecuada en la sociedad. Como resultado de la evaluación, las familias que satisfagan todas las necesidades de antemano establecidas son calificadas no pobres y las restantes como pobres.

\section{Medición según el concepto de pobreza relativa}

Las líneas de pobreza bajo el concepto de pobreza relativa se definen en relación con la distribución global del ingreso o del consumo del país. Por ejemplo, puede fijarse la línea de pobreza relativa en $40 \%$ del ingreso o del consumo medio del país objeto de estudio, en cuyo caso, quienes dispongan de un ingreso inferior a 40\% del ingreso medio serán calificados como pobres

\section{- Líneas de pobreza absoluta y relativa}

La línea de pobreza absoluta es fija, ya sea que se use el concepto de subsistencia o de necesidades básicas insatisfechas. Si se aplica una línea de pobreza absoluta los criterios, parámetros y valores se mantienen invariables conforme transcurre el tiempo, mientras que la línea de pobreza relativa considera que las personas tienden a percibir su propio bienestar o posible situación de pobreza en función del bienestar de los demás. En consecuencia el umbral de pobreza no es constante en el tiempo, sino por el contrario, cambiante, para que tome en cuenta las necesidades que surgen con los nuevos servicios y las nuevas actividades de participación en la sociedad.

Según Céspedes y Jiménez (2006), el INEC por ejemplo utiliza la línea de pobreza absoluta, ya que el costo de la línea de pobreza es el correspondiente al año 1995 y lo mantiene constante, y solo actualiza su costo por efectos de la inflación, pero el contenido de la canasta (lista de bienes y cantidad de cada uno) se mantiene igual de un año a otro.

\section{-Índice de Sen}

Presenta tres componentes: el Indice de Gini, que se utiliza para medir la desigualdad en los ingresos, el porcentaje de pobres en la línea de pobreza y la magnitud de la pobreza.

\section{-Indice de Desarrollo Humano}

Es el indicador utilizado por el Programa de las Naciones Unidas para el Desarrollo (PNUD, 1990) y se calcula con base en tres indicadores claves: esperanza de vida, nivel de educación e ingreso. Se toman en cuenta otros aspectos que atañen al desarrollo humano como son: derechos humanos, crecimiento económico, globalización, adelanto tecnológico, participación comunitaria.

\section{El campo de fuerzas en los estudios sobre la pobreza en Costa Rica en los últimos 10 años}

En el nivel del campo simbólico de los estudios sobre la pobreza, existe una posición hegemónica de uno de los conceptos de pobreza.

En el caso costarricense, a partir de la imposición del Consenso de Washington, se empezó a sustituir al estructuralismo "cepalino"en la conciencia de nuestros intelectuales, por un discurso neoliberal. 
Se generó un discurso hegemónico que le atribuye al crecimiento económico un papel crucial para la reducción de la pobreza, concibiéndolo como generador de oportunidades de incorporación individual al mercado, además de la reducción de la responsabilidad estatal y la apertura la descentralización y la necesidad de un nuevo papel de las organizaciones de la sociedad civil.

Muchos investigadores, políticos, tecnócratas iniciaron sus discursos alrededor de la necesidad de que Costa Rica se convirtiera en una sociedad de libre mercado, abierta a los flujos económicos globales, que coincidía abiertamente con las exigencias de los organismos financieros internacionales.

El grupo de "los liberales" que venían aglutinados básicamente en la Asociación Nacional de Fomento Económico (ANFE) desde hacía años, convergieron en los paradigmas o recetas del Fondo Monetario Internacional (FMI) y del Banco Mundial y vieron así fortalecidas sus viejas posiciones anti-estatistas y pro-mercado.

Los intelectuales socialdemócratas y de izquierda en general, no tuvieron mucha oportunidad de expresar sus planteamientos opuestos, ya que la economía recesiva del 78 al 82, necesitaba en parte de las recetas del FMI. Los genuinos socialdemócratas aglutinados en el Partido Liberación Nacional se vieron afectados por la crisis de la socialdemocracia en Europa reflejada en severas reformas de los sistemas de pensiones y prestaciones sociales a cargo del Estado y no pudieron articular un discurso propio.

Para la izquierda, la debacle institucional del comunismo en Europa, completó un escenario de "pérdida de vigencia". A esto hay que agregar que a partir de ese momento, los ministros de Hacienda y Presidentes del Banco Central, no importando el partido que gobernara, han sido correligionarios del FMI y de los ajustes estructurales pues ello significaba un requerimiento tácito para una "buena interlocución" por parte de este organismo sobre los endeudados gobiernos, desde entonces.
En la década de los 90, los viejos problemas de pobreza se agudizaron, aumenta la desigualdad y exclusión social, según lo establece el X Informe del Estado de la Nación. Las consecuencias de los procesos de estabilización y ajuste estructural, obligaron a que las propias agencias financieras internacionales reconocieran la necesidad de realizar una reforma social para hacerles frente.

Es en los informes posteriores a 1990, donde el Banco Mundial plantea de manera directa estrategias para la contención de los procesos de ajuste y superación de la pobreza. Reconoce la necesidad de disminuir la desigualdad social, ya que ésta tiende a limitar el crecimiento económico, y solicita el compromiso de los gobiernos de lograr un crecimiento sostenido para reducir la vulnerabilidad social ${ }^{1}$ y la exclusión social ${ }^{2}$.

Plantea además otras medidas colaterales como la gobernabilidad de los procesos de ajuste, la necesidad de replantear las instituciones de seguridad social para incrementar los niveles de ahorro interno y la urgencia de flexibilizar los mercados laborales.

Todas estas recomendaciones fueron acogidas por los gobiernos costarricenses.

1. Vulnerabilidad es el nivel de riesgo que afronta una familia o individuo a perder la vida, sus bienes y propiedades, y su sistema de sustento (esto es, su medio de vida) ante una posible catástrofe. Dicho nivel guarda también correspondencia con el grado de dificultad para recuperarse después de tal catástrofe (Pérez, s.f). La vulnerabilidad tiene por tanto dos partes: una parte externa, de los riesgos, convulsiones y presión a la cual está sujeto un individuo o familia; y una parte interna, que es la indefensión, esto es, una falta de medios para afrontar la situación sin pérdidas perjudiciales, ante los procesos de desastre y la posterior rehabilitación.

2. Exclusión social es el proceso mediante el cual los individuos o grupos son total o parcialmente excluidos de una participación plena en la sociedad en la que viven". Tal proceso, opuesto al de "integración social", da lugar a una privación múltiple, que se manifiesta en los planos económico, social y político. http://dicc.hegoa.efaber.net/listar/mostrar/68 
En 2004, inspirado en la relaciones conceptuales entre desarrollo y libertad propuestas por Sen, el PNUD (2004) publicó el informe La democracia en América Latina. Hacia una democracia de ciudadanos y ciudadanas, a partir de los insumos teóricos de Guillermo O.Donnell. En este estudio el PNUD realiza un balance en la región desde una concepción de ciudadanía integral, incorporando el pleno reconocimiento de las ciudadanías civil, política y social, y concluye que el principal desafío de las democracias latinoamericanas lo constituyen los déficits en ciudadanía social.

En cuanto a la producción documental costarricense, CEPAL-CLADES (1995) menciona que en Costa Rica se realizaron innumerables trabajos, concentrados alrededor de cuatro grandes ejes:

1. Los costos sociales del ajuste, gasto social y reformas a la seguridad social.

2. El aumento de la pobreza, con estudios concretos y focalizados para su disminución.

3. Empleo y desempleo.

4. Desempeño de los servicios sociales en el contexto de la crisis y el ajuste de las propuestas de descentralización. En este campo específico, los esfuerzos de analistas, fueron aislados, con diseños tímidos sobre aspectos micro del régimen municipal, sin ninguna factibilidad, ya que no existe voluntad política para la descentralización.

Entre los trabajos más representativos están los ligados con: conceptos de pobreza y métodos para medirla, los relacionados con el uso del gasto público y las políticas tributarias para el desarrollo humano, la equidad social y la eficacia; también los asociados a las políticas públicas para aminorar la pobreza. Un grupo de las investigaciones se relaciona con los posibles impactos de la pobreza a partir de la inserción en el mercado mundial, la globalización y la participación en organizaciones del comercio mundial.
Otro grupo de investigaciones apuntan a temas como ciudadanía, derechos humanos y democracia para observar si con la pobreza, la exclusión y la desigualdad se dan oportunidades para desarrollar estas capacidades.

Cabe mencionar que el Centro de Investigaciones Históricas de América Central de la Universidad de Costa Rica, en su Programa de Investigación en Historia Económica y Social, abordó el tema sobre la Pobreza e Historia en Costa Rica desde 1830 hasta 1950 desde diversas facetas como son: las percepciones y representaciones sociales, las formas mendicantes y filantrópicas, las luchas sociales, la vivienda, perfiles socioeconómicos, costo y condiciones de vida. ${ }^{3}$

\section{La necesidad de una apertura a una fase multiparadigmática en los estudios de pobreza en Costa Rica}

Los estudios y reuniones internacionales promovidos por el PNUD, los informes de la CEPAL, así como la producción de nuevos indicadores para medir la pobreza y el desarrollo humano avanzan sobre una concepción del bienestar articulada a las ideas de igualdad de oportunidades, calidad de vida, derechos ciudadanos, especialmente los civiles, humanos y sociales (CEPAL 2010 que en su anuario estadístico tiene informes para todos los años).

Barba (2009), Ivo (2004) y Sojo (2003) argumentan que un reto evidente de la producción documental sobre la pobreza en América Latina, es trascender la tendencia a considerar que la reducción de la pobreza y el crecimiento económico, son esferas distintas con respecto al

3. Entre sus autores están Ronny Viales, Ana Paulina Malavassi, Vladimir de La Cruz, Juan José Marín, Miguel Guzmán, Manuel Chacón, Hilda Bonilla, Olga Marta Ramírez, Enmanuel Barrantes, Ana María Botey, William Elizondo y Javier Rodriguez. 
tema de la ciudadanía y los derechos sociales económicos y culturales. Asimismo, los autores analizan como positiva la tendencia de ver la redefinición de la pobreza como un problema que incluye dimensiones políticas, culturales, derechos y capacidades. En esta línea el planteamiento de varios autores es proponer no separar los aspectos económicos y sociales, sino que la pobreza debe ser vista como eje del desarrollo, y que las políticas públicas deben de integrar los procesos de integración y bienestar social con el desempeño del mercado, por medio de la producción y distribución de riqueza; y el potencial solidario del capital social.

La CEPAL es una de las organizaciones regionales que ha planteado enfoques alternativos sobre la pobreza. Su propuesta más destacada es solicitar a los países una transformación productiva con equidad social. Plantea la noción de calidad del crecimiento y el rechazo a la separación de tareas entre la política social y la política económica. Además, considera necesario, el impulso y promoción de los derechos económicos, sociales y culturales, de carácter universal e indivisible, que pongan en la agenda social la construcción de la ciudadanía social y rompan con la oposición entre universalidad y focalización, que ha sido el norte de las políticas sociales en América Latina en las últimas décadas ${ }^{4}$.

El análisis de la pobreza y su combate, desde el punto de vista institucional y gerencial, es poco

4. La CEPAL, indica que los países de la región no solo enfrentan hoy los mayores retos de la historia en términos de pobreza, desigualdad y exclusión social, sino crecientes problemas de desempleo, subempleo, informalidad laboral, desigualdades y exclusiones por razones de raza, género y edad, inseguridad ciudadana. (CEPAL 2001; CEPAL/UNICEF 2001; Gacitúa, Sojo y Davis de la CEPAL.), así como la producción de nuevos indicadores para medir la pobreza, el desarrollo humano, etc; que avanzan una concepción del bienestar articulada a las ideas de igualdad de oportunidades, calidad de vida, derechos ciudadanos, especialmente los civiles, humanos y sociales desarrollada y existen pocos trabajos. En América Latina reconocemos los trabajos de Bernardo Kliskberg, y en Costa Rica, Johnny Meoño 5 .

Para Costa Rica, a mediados de los 80, se inicia en el país un trabajo sistemático que permite generar información con algún grado de confiabilidad sobre la dimensión de la pobreza y su localización. Al respecto los investigadores que más han trabajado el tema se han ubicado dentro de los paradigmas universalista o residual. En el primero está, sin duda, Juan Diego Trejos, del Instituto de Investigaciones en Ciencias Económicas de la Universidad de Costa Rica, en el segundo Victor Hugo Céspedes y Ronulfo Jiménez, y la Academia de Centro América.

El Instituto Nacional de Estadística y Censos (INEC), que realiza la Encuesta Nacional de Hogares, el Instituto Mixto de Ayuda Social (IMAS) que aplica la metodología de Línea de Pobreza, el Proyecto Estado de la Nación que mide el Indice de Desarrollo Humano, presentan ambivalencias y contradicciones ya que por un lado su interpretación de la pobreza y la medición que realizan parten de la perspectiva de problemas para la satisfacción o privación de necesidades básicas, mientras que el discurso social de los últimos gobiernos ha sido netamente neoliberal y la jerarquía de estas organizaciones oscila entre el paradigma residual y el universalista.

En 2002, el rector de la Universidad de Costa Rica, Gabriel Macaya, nombró una Comisión para orientar al gobierno en su lucha contra la pobreza. Los aportes de la Comisión, constituyeron un hito analítico-interpretativo porque rompió con la unilateralidad disciplinaria y conceptual predominante en los estudios, al reconocer e incorporar en su análisis los aspectos de "gobernabilidad" o gestión pública determinantes

5. Se trata de los artículos de Kliksberg B (2002) "Hacia una economía con rostro humano", y de Meoño J (2008) Descentralización integral para el desarrollo de Costa Rica. 
de la dinámica del desarrollo del país. La omisión de estos temas ha ocasionado negligencia e improvisación en la eficacia para el ejercicio de las normas constitucionales y legales superiores que tiene nuestra Constitución y en la lucha contra la pobreza. ${ }^{6}$

\section{CONCLUSIONES}

Se puede afirmar que a pesar de que existe acuerdo acerca de que el fenómeno de la pobreza tiene un carácter estructural, no hay un diagnóstico igualmente compartido sobre sus causas. En pocos temas como en el de la pobreza, la reflexión científica ha estado marcada por connotaciones políticas. La pobreza en sí misma conlleva una dimensión política, ya que los intereses de los diferentes grupos tienen una fuerte influencia en los modelos de distribución y en la existencia misma de la pobreza. Ahondar en las raíces de la pobreza supone plantear cuestiones difíciles y conflictivas, lo que explica las reticencias y los rechazos que acompañan el proceso del conocimiento sobre el tema.

Por ello, en el análisis de la pobreza las posiciones de partida han marcado decisivamente el concepto y el diagnóstico. En un extremo se encuentran imaginarios sociales que parten de considerar a la pobreza como un fenómeno natural de la condición humana, de carácter fatalista, como si fuera una enfermedad heredada que aún no tiene cura. En el otro extremo se halla la posición de que la pobreza es un fenómeno que solo puede explicarse en el marco de las reglas de funcionamiento del modelo económico actual de carácter globalizador, que no plantea su erradicación como objetivo.

6. Se trata del libro "Hacia un enfoque integral de la pobreza", donde participaron los profesores de la Facultad de Ciencias Económicas: Johnny Meoño Segura, Juan Diego Trejos Solorzáno, Edgar Gutiérrez Espeleta y Mayela Cubillo Mora.
Históricamente, se ha enfatizado más la precisión y exactitud de la medición de la pobreza que su concepto. En otras palabras, la preocupación principal de los autores de los estudios sobre la pobreza ha sido marcada por la medición, privilegiando los datos duros. ¿Cuántos hay? ¿Dónde están? ¿Quiénes son pobres? ¿Cuáles son las condiciones imprescindibles para que no mueran? ¿Cuáles son los riesgos y vulnerabilidades que enfrentan? Esto ha condicionado los esfuerzos por conceptualizarla, hasta el punto que se considera que se profundiza más y mejor en el conocimiento de la misma, cuanto más se la pudiera cuantificar de forma más precisa.

El predominio de la medición sobre lo conceptual no es casual, ya que la hegemonía del enfoque de subsistencia en la concepción de pobreza reduce los elementos definitorios de la pobreza fundamentalmente al ingreso, estableciendo el umbral o límite al mínimo de las necesidades para subsistir. Este umbral si bien es nítido y medible es poco exigente éticamente.

Los organismos financieros siguen utilizando la concepción de pobreza como satisfacción de necesidades básicas, concepción que es seguida por nuestras organizaciones y operadores de la política social. El Banco Mundial, por ejemplo, sigue estableciendo el ingreso de un dólar-día por persona como referente de la pobreza, con lo que apenas se ha modificado su concepto original, que entiende ésta desde un referente absoluto, como mera supervivencia biológica referida a los mínimos de supervivencia. El pobre es visto entonces como un indicador negativo del progreso social.

Cuanto más se restringe el concepto de pobreza a un ingreso insuficiente para cubrir los bienes y servicios individuales de primera necesidad, e incluso a los bienes colectivos y o servicios públicos, más fácil resulta argumentar que para superar el fenómeno lo único que se requiere es un crecimiento nacional de la riqueza material. 
En contraste, si asumimos, como Amartya Sen, que la pobreza o calidad de vida se encuentra en las capacidades de las personas, el objetivo prioritario de la sociedad es asegurar la construcción de esas capacidades. Formulada de esta manera la pobreza implica establecer qué capacidades básicas y qué funcionamientos son los realmente necesarios y valiosos para que las personas se realicen como tales. Se introduce así otros elementos de la pobreza que habitualmente quedan fuera de las propuestas: el valor de la autonomía, la responsabilidad, la libertad, la capacidad de acción y comunicación, como activos requeridos por los pobres.

El abordaje de la pobreza debería darse también desde un enfoque cualitativo y no solo cuantitativo. Es necesario ampliar la caracterización de los pobres, incorporando los sentidos o significados que se producen dentro de la pobreza. Es decir, estudiarla desde su propio entorno natural, dar relevancia a los aspectos subjetivos de la conducta humana sobre las características objetivas, explorar los significados y el uso del lenguaje simbólico, más que de los signos numéricos (estadística), estudiar las implicaciones subjetivas de las personas pobres, elaborar biografías, itinerarios, proyectos, estrategias, utilizar otras técnica como: grupos focales, entrevistas, historias de vida, representaciones sobre el poder y la identidad nacional, entre otros.

Por lo anterior, se puede concluir que en el país, es necesario, realizar estudios con enfoques multidimensionales que aborden la pobreza. Que las políticas públicas de desarrollo se formulen articulando las esferas económicas, políticas y sociales, buscando como fines la protección de los derechos de las personas, que permitan generar ciudadanía y gobernabilidad en el marco de la globalización.

Es necesario también, investigaciones sistémicas de la seguridad social en el país, para analizar la hibridación de los modelos universales con los focalizados, tan utilizados por nuestros gobiernos. Es importante también asumir el estudio de los procesos de transición del dominio de un paradigma a otro y analizar su concreción institucional, es decir cuál es el manejo de los operadores de las políticas sociales, como son el Instituto Mixto de Ayuda Social, el Fondo Nacional de Asignaciones Familiares (FODESAF), entre otros; y como ejecutan su actividades cuando se pasa de un enfoque a otro.

Es necesario además, enfoques interdisciplinarios que profundicen en la relación entre desigualdad y pobreza en las diferentes regiones del país, y cómo contrastarlo con el discurso cotidiano de que somos una sociedad igualitaria, y analizar la manera en que es abordada la pobreza en las representaciones sociales e incorporar este elemento sociocultural en las acciones de superación de la pobreza.

Se hace necesario reconsiderar las viejas expresiones del contrato social, reformular la definición de lo justo y lo equitativo, y reinventar las formas de la solidaridad. La mirada de la pobreza como violación a los Derechos Humanos permite ampliar el enfoque de la pobreza y situarlo en una perspectiva filosófica, y el concepto de protección social como un componente de una visión más holística de manejo social del riesgo frente a la vulnerabilidad. Concepciones para mirar la pobreza que evidentemente no se están haciendo en el país, por lo tanto, las políticas sociales actuales omiten o abordan sin conocimiento de fondo ni visión ni formación, echando a perder mucho de lo bueno que ha surgido en cuanto a "modelos de política social".

Finalmente, un tema de importancia estratégica para combatir la hegemonía de los imaginarios, discursos y paradigma dominantes. ¿Cómo construir consensos, coaliciones sociales y políticas que puedan impulsar una imagen país, una 
agenda social más democrática e inclusiva que le de sustento histórico a nuevos paradigmas de bienestar social?, como lo fue en el pasado (década de los 40) la creación del estado de Bienestar anclados básicamente en una coalición formada entre el Partido Comunista, la Iglesia Católica y el partido político dirigido por el Dr. Calderón Guardia (Calderonismo).

\section{REFERENCIAS}

Atkinson, A. \& Bourguignon, F. (1999). Poverty and Inclusion from a World Perspective. Paris: Paper presented at the Annual Bank Conference on Development Economics.

Barba, C. (2009). Los estudios de pobreza en América Latina. Revista Mexicana de Sociologia, 71, edición especial, 9-49.

Bourdieu, P. (1991). Razones prácticas sobre la teoría de la acción. Barcelona. Anagrama.

Beccaria, L., Feres, J.C., Sainz, P. (s.f.). Comisión Económica Para América Latina (CEPAL). Recuperado el 09-012011 de: www.eclac.cl/deype/mecovi/taller4.ht

Banco Mundial (2001). Informe Sobre el Desarrollo Mundial 2000/2001. Lucha contra la pobreza. Washington.

Castoriadis, C. (1986). Lo imaginario: la creación en el dominio histórico-social. En Los Dominios del Hombre. Las encrucijadas del laberinto. Barcelona: Gedisa.

CEPAL- Centro Latinoamericano de Documentación Económica y Social (CLADES) (1995). Políticas Sociales: Resúmenes de documentos. Serie INFOPLAN, N.10. Santiago de Chile: Publicaciones de las Naciones Unidas.

CEPAL (2010). Anuario estadístico de América Latina y el Caribe. Recuperado el 10-01-2011 de www.eclac.org/

Céspedes, V.H. \& Jiménez, R. (2006). Pobreza en Costa Rica. San José: Academia de Centro América.

Cortina, A. (2009). Pobreza y libertad. Erradicar la pobreza desde el enfoque de Amartya Sen. Madrid: Tecnos..

Programa Estado de la Nación (2009). Décimo Informe Estado de la Nación en Desarrollo Humano Sostenible. San José, Programa Estado de la Nación.

Esping-Andersen, G. (1987). The comparison of policy regimes: En: En Esping-Andersen, Gosta Rein, Martin y Lee Rainwater (Eds.). Stagnation and Renewal in Social
Policy. The rise and fall of policy regimes. New York: M.E. Sharpe, Inc.

Foucault, M. (1984). La Arqueología del Saber. México: Siglo $X X I$.

Instituto Nacional de Estadística y Censos (INEC) (2010). http://www.inec.go.cr/Web/Home/pagPrincipal. aspx\#

IVO (Instituto de Estudios para el desarrollo) (2004). Democracia, desigualdad e capital social en América Latina". en Integración, identidade e capital social. Brasilia.

Kliksberg, B. (2002) Hacia una economia con rostro humano. Revista Venezolana de Gerencia, 17 (19). Maracaibo: Universidad de Zulia,.

Kuhn, T. (1987). La estructura de las revoluciones científicas. F.C.E. México

Marshall, T.H. (1964): Welfare in the Context of Social Policy. En Marshall, T.H. (1981): The Right to Welfare and other Essays. Heineman, Londres

Meoño, J. (2008). Descentralización integral para el desarrollo de Costa Rica. Un necesario diagnóstico modelístico. En Revista de Ciencias Económicas, 26 (1) Universidad de Costa Rica.

O'Donnell, G. (2008). Algunas reflexiones acerca la democracia, el Estado y sus múltiples caras. En XIII Congreso Internacional del CLAD sobre la Reforma del Estado y de la Administración Pública, Buenos Aires, Argentina.

Pérez, K. (s. f). Vulnerabilidad. Diccionario de Acción Humanitaria y Cooperación al Desarrollo. Recuperado el 02-01-2011 de: http://dicc.hegoa.efaber.net/listar/ mostrar/228

Programa de las Naciones Unidas para el Desarrollo (PNUD) (1990). Concepto y medición de desarrollo humano. PNUD. New York

PNUD (2004) La democracia en América Latina Hacia una democracia de ciudadanas y ciudadanos. New York

Rowntree, S. (1901). Poverty, A study of Town life. Mc Milliam. Londres.

Sen, A. (1995). Nuevo examen de la desigualdad. Madrid, España: Alianza Economía.

Sen, A. y Dréze, J. (1989). Hunger an Public Action. Oxford: Claredon Pres. Londres 
RNA Revista Nacional de Administración

Sojo, C. (2001). The socio-political and cultural dynamics of social exclusion. En Gacitua, E., Sojo, C., \& Davis, S.(Eds.). Social Exclusion and Poverty Reduction in Latin America and the Caribbean. San José, Costa Rica: Banco Mundial y FLACSO.

Sojo, C. (2002). Desarrollo social en América Latina, temas y desafíos para las políticas públicas. San José: FLACSO y Banco Mundial

Townswend, P. (1993). The International Analysis of Poverty. Londres: Harvester Wheatsheaf.

Recibido: 31 de enero de 2011 Aceptado: 15 de marzo de 2011 